\title{
A New Full-NT-Step Infeasible Interior-Point Algorithm for SDP Based on a Specific Kernel Function
}

\author{
Samir Bouali, Samir Kabbaj \\ Department of Mathematics and Informatics, Faculty of Sciences, \\ University Ibn Tofail, Kenitra, Morocco \\ Email: samir_bouali34@yahoo.fr, samkabbaj@yahoo.fr
}

Received June 7, 2012; revised August 7, 2012; accepted August 15, 2012

\begin{abstract}
In this paper, we propose a new infeasible interior-point algorithm with full NesterovTodd (NT) steps for semidefinite programming (SDP). The main iteration consists of a feasibility step and several centrality steps. We used a specific kernel function to induce the feasibility step. The analysis is more simplified. The iteration bound coincides with the currently best known bound for infeasible interior-point methods.
\end{abstract}

Keywords: Semidefinite Programming; Full Nesterov-Todd Steps; Infeasible Interior-Point Methods; Polynomial Complexity; Kernel Functions

\section{Introduction}

In this paper we deal with SDP problems, whose primal and dual forms are:

$$
(P) \min \left\{\operatorname{Tr}(C X): \operatorname{Tr}\left(A_{i} X\right)=b_{i}, i=1,2, \cdots, m, X \succeq 0\right\},
$$

and

$$
\text { (D) } \max \left\{b^{T} y: \sum_{i=1}^{n} y_{i} A_{i}+S=C, S \succeq 0\right\},
$$

where $C, X, S, A_{i} \in \mathbb{S}^{n}, \quad b, y \in \mathbb{R}^{m}$. The matrices $A_{i}$, $i=1,2, \cdots, m$ are assumed to be linearly independent.

The use of Interior-Point Methods (IPMs) based on the kernel functions becomes more desirable because of the efficiency from a computational point of view. Many researchers have been attracted by the Primal-Dual IPMs for SDP. For a comprehensive study, the reader is referred to Klerk [1], Roos [2] and Wolkowicz et al. [3]. Bai et al. [4] introduced a new class of so-called eligible kernel functions for Linear Optimization (LO) which are defined by some simple properties following the same way of Peng et al. who have designed a class of IPMs based on a so-called self-regular proximities [5]. These methods use the new search directions which are different than the classic Newton directions. Some extensions were successfully made by Mansouri and Roos [6], Liu and Sun [7]. In the current paper, we propose a new infeasible interior-point algorithm, whose feasibility step is induced by a specific kernel function.

In the sequel, we denotes $e$ as the all one vector and $\lambda(X)$ the vector of eigenvalues of $X \in \mathbb{S}^{n}$. Two dif- ferent forms of norm will be used

$$
\|X\|_{2}:=\max _{i}\left\{\left|\lambda_{i}(X)\right|\right\},\|X\|_{F}=\sqrt{\sum_{i=1}^{n} \lambda_{i}^{2}(X)} .
$$

\section{The Statement of the Algorithm}

We start usually with assuming that the initial iterates $X^{0}, y^{0}$ and $S^{0}$ are as follows

$$
X^{0}=S^{0}=\zeta I, y^{0}=0, \mu^{0}=\zeta^{2},
$$

where $I$ is the $n \times n$ identity matrix, $\mu^{0}$ is the initial dual gap and $\zeta>0$ is such that

$$
\left\|X^{*}+S^{*}\right\|_{2} \leq \zeta,
$$

for some optimal solution $\left(X^{*}, y^{*}, S^{*}\right)$ of $(P)$ and (D).

\subsection{The Feasible SDP Problem}

The perturbed KKT condition for $(P)$ and $(D)$ is

$$
\begin{gathered}
\operatorname{Tr}\left(A_{i} X\right)=b_{i}, \quad i=1,2, \cdots, m, X \succeq 0, \\
\sum_{i=1}^{n} y_{i} A_{i}+S=C, S \succeq 0 \\
X S=\mu I .
\end{gathered}
$$

Based on different symmetrization schemes, several search directions have been proposed.

As in Mansouri and Roos [6], we use in this paper, the so-called NT-direction determined by the following system 


$$
\begin{gathered}
\operatorname{Tr}\left(A_{i} \Delta X\right)=0, i=1,2, \cdots, m, \\
\sum_{i=1}^{n} \Delta y_{i} A_{i}+\Delta S=0, \\
\Delta X+P \Delta S P^{T}=\mu S^{-1}-X .
\end{gathered}
$$

where

$$
P=X^{\frac{1}{2}}\left(X^{\frac{1}{2}} S X^{\frac{1}{2}}\right)^{-\frac{1}{2}} X^{\frac{1}{2}}=S^{-\frac{1}{2}}\left(S^{\frac{1}{2}} X S^{\frac{1}{2}}\right) S^{-\frac{1}{2}}
$$

We also define the square root matrix $D=P^{\frac{1}{2}}$.

The matrix $D$ can be used to rescale $X$ and $S$ to be the same matrix $V$, defined by

$$
V:=\frac{1}{\sqrt{\mu}} D^{-1} X D^{-1}=\frac{1}{\sqrt{\mu}} D S D .
$$

It is clear that $D$ and $V$ are symmetric and positive definite. Let us further define

$$
\begin{aligned}
& D_{X}:=\frac{1}{\sqrt{\mu}} D^{-1} \Delta X D^{-1}, \\
& D_{S}:=\frac{1}{\sqrt{\mu}} D \Delta S D \text { and } \bar{A}_{i}:=\frac{1}{\sqrt{\mu}} D A_{i} D,
\end{aligned}
$$

where $i=1,2, \cdots, m$. Using the above notations, the third equation of the system (1) is then formulated as follows

$$
D_{X}+D_{S}=V^{-1}-V \text {. }
$$

It is clear that the first two equations imply that $D_{X}$ and $D_{S}$ are orthogonal, i.e. $\operatorname{Tr}\left(D_{X} D_{S}\right)=0$, which yields that $D_{X}$ and $D_{S}$ are both zero if and only if $V^{-1}-V=0$. In this case, $X$ and $S$ satisfy $X S=\mu I$, implying that $X$ and $S$ are the $\mu$-centers. Hence, we can use the norm $\left\|V^{-1}-V\right\|_{F}$ as a quantity to measure closeness to the $\mu$-centers. Let us define

$$
\delta(X, S ; \mu):=\delta(V):=\frac{1}{2}\left\|V^{-1}-V\right\|_{F}
$$

\subsection{The Perturbed Problem}

For any $v$ with $0<v \leq 1$, we consider the perturbed problem $\left(P_{v}\right)$, defined by

$$
\begin{gathered}
\left(P_{v}\right) \min _{X} \operatorname{Tr}\left(\left(C-v R_{C}^{0}\right) X\right) \\
\text { st }: \operatorname{Tr}\left(A_{i} X\right)=b_{i}-v\left(r_{b}^{0}\right)_{i}, i=1,2, \cdots, m, X \succeq 0,
\end{gathered}
$$

and its dual problem $\left(D_{v}\right)$ given by

$$
\begin{gathered}
\left(D_{v}\right) \max _{y, S} \sum_{i=1}^{n}\left(b_{i}-v\left(r_{b}^{0}\right)_{i}\right) y_{i} \\
\text { st : } \sum_{i=1}^{n} y_{i} A_{i}+S=C-v R_{C}^{0}, S \succeq 0
\end{gathered}
$$

where

$$
\begin{aligned}
& \left(r_{b}^{0}\right)_{i}=b_{i}-\operatorname{Tr}\left(A_{i} X^{0}\right), \\
& R_{C}^{0}=C-\sum_{i=1}^{n} y_{i}^{0} A_{i}-S^{0} .
\end{aligned}
$$

Note that if $v=1$ then $X=X^{0}$ and $(y, S)=\left(y^{0}, S^{0}\right)$, yielding that both $\left(P_{v}\right)$ and $\left(D_{v}\right)$ are strictly feasible.

Lemma 1 ([6], Lemma 4.1) Let the original problems, $(P)$ and $(D)$, be feasible. Then for each $v$ such that $0<v \leq 1$ the perturbed problems $\left(P_{v}\right)$ and $\left(D_{v}\right)$ are strictly feasible.

We assume that $(P)$ and $(D)$ are feasible. It follows from Lemma (1) that the problems $\left(P_{v}\right)$ and $\left(D_{v}\right)$ are strictly feasible. Hence their central path exists. The central path of $\left(P_{v}\right)$ and $\left(D_{v}\right)$ is defined by the solution sets $\{(X(\mu), y(\mu), S(\mu)), \mu>0\}$ of the following system

$$
\begin{gathered}
\operatorname{Tr}\left(A_{i} X\right)=b_{i}-v\left(r_{b}^{0}\right)_{i}, i=1,2, \cdots, m, X \succeq 0, \\
\sum_{i=1}^{n} y_{i} A_{i}+S=C-v R_{C}^{0}, S \succeq 0 \\
X S=\mu I .
\end{gathered}
$$

If $v \in(0,1]$ and $\mu=v \zeta^{2}$, we denote this unique solution as $(X(v), y(v), S(v)) . X(v)$ is the $\mu$-center of $\left(P_{v}\right)$, and $(y(v), S(v))$ the $\mu$-center of $\left(D_{v}\right)$. By taking $v=1$, one has

$(X(1), y(1), S(1))=\left(X^{0}, y^{0}, S^{0}\right)=(\zeta I, 0, \zeta I)$. Initially, one has $X=S=\zeta I$ and $\mu=\zeta^{2}$, whence $V=I$ and $\delta(X, S ; \mu)=0$. In what follows, we assume that at the start of each iteration, $\delta(X, S ; \mu)$ is smaller than a threshold value $\tau$ which is obviously true at the start of the first iteration. The following system is used to define the step $\left(\Delta^{f} X, \Delta^{f} y, \Delta^{f} S\right)$

$$
\begin{aligned}
& \operatorname{Tr}\left(A_{i} \Delta^{f} X\right)=\theta v\left(r_{b}^{0}\right)_{i}, X \succeq 0, \\
& \sum_{i=1}^{n} \Delta^{f} y_{i} A_{i}+\Delta^{f} S=\theta \nu R_{C}^{0}, S \succeq 0 \\
& \Delta^{f} X+P \Delta^{f} S P^{T}=(1-\theta) \mu S^{-1}-X .
\end{aligned}
$$

where $\theta \in(0,1)$ and $i=1,2, \cdots, m$.

Inspired by [8], we used in the third equation for the above system, a linearization $X^{f} S^{f}=(1-\theta) \mu I$, which means that we target the $\mu^{+}$-center of $\left(P_{v^{+}}\right)$and $\left(D_{v^{+}}\right)$.

After the feasibility step, the new iterates are given by $X^{f}=X+\Delta^{f} X, \quad y^{f}=y+\Delta^{f} y$ and $S^{f}=S+\Delta^{f} S$. The algorithm begins with an infeasible interior point $(X, y, S)$ such that $(X, y, S)$ is feasible for the perturbed problems, $\operatorname{Tr}(X S)=n \mu$ and $\delta(X, S ; \mu) \leq \tau$. First we find a new point $\left(X^{f}, y^{f}, S^{f}\right)$ which is feasible for the perturbed problems with $v^{+}:=(1-\theta) v$. Then $\mu$ is decreased to $\mu^{+}:=(1-\theta) \mu$. A few centering 
steps are applied to produce new points $\left(X^{+}, y^{+}, S^{+}\right)$ such that $\delta\left(X^{+}, S^{+} ; \mu^{+}\right) \leq \tau$. This process is repeated until the algorithm terminates. Starting at the iterates $\left(X^{f}, y^{f}, S^{f}\right)$ and targeting the $\mu$-center, the centering steps are obtained by solving the system (1).

\subsection{Infeasible IPMs Based on a Specific Kernel Function}

Now we introduce the definition of a kernel function. We call $\psi:(0, \infty) \rightarrow[0, \infty)$ a kernel function if $\psi$ is twice differentiable and the following conditions are satisfied

1) $\psi^{\prime}(1)=\psi(1)=0$;

2) $\psi^{\prime \prime}(t)>0$ for all $t>0$;

3) $\lim _{t \downarrow 0} \psi(t)=\lim _{t \rightarrow \infty} \psi(t)=\infty$.

We define

$$
\tilde{V}:=\frac{V}{\sqrt{1-\theta}}, \tilde{D}_{X}:=\frac{D_{X}}{\sqrt{1-\theta}} \text { and } \tilde{D}_{S}:=\frac{D_{S}}{\sqrt{1-\theta}} .
$$

By using the scaled search directions $D_{X}^{f}$ and $D_{S}^{f}$ as defined in (4), the system (7) can be reduced to

$$
\begin{gathered}
\operatorname{Tr}\left(\bar{A}_{i} D_{X}^{f}\right)=\frac{1}{\mu} \theta v\left(r_{b}^{0}\right)_{i}, i=1,2, \cdots, m, X \succeq 0, \\
\sum_{i=1}^{n} \Delta^{f} y_{i} \bar{A}_{i}+D_{S}^{f}=\frac{1}{\sqrt{\mu}} \theta v D R_{C}^{0} D, S \succeq 0 \\
D_{X}^{f}+D_{S}^{f}=\sqrt{1-\theta}\left(\tilde{V}^{-1}-\tilde{V}\right) .
\end{gathered}
$$

According to (8), Equation (9) can be rewritten as

$$
\tilde{D}_{X}^{f}+\tilde{D}_{S}^{f}=\tilde{V}^{-1}-\tilde{V} .
$$

It is clear that the right-hand side of the above equation is the negative gradient direction of the following barrier function $\Psi(V):=\operatorname{Tr}(\psi(\tilde{V}))$ whose kernel logarithmic barrier function is $\psi(t)=\frac{1}{2}\left(t^{2}-1\right)-\log (t)$. Therefore, the aforementioned equation can be rewritten as

$$
\tilde{D}_{X}^{f}+\tilde{D}_{S}^{f}=-\nabla \Psi(\tilde{V}) .
$$

Inspired by the work of $[4,7,9]$, and by making a slight modification of the standard Newton direction, the new feasibility step used in this paper, is defined by the following different system:

$$
\begin{gathered}
\operatorname{Tr}\left(\bar{A}_{i} D_{X}^{f}\right)=\frac{1}{\mu} \theta v\left(r_{b}^{0}\right)_{i}, i=1,2, \cdots, m, X \succeq 0, \\
\sum_{i=1}^{n} \Delta^{f} y_{i} \bar{A}_{i}+D_{S}^{f}=\frac{1}{\sqrt{\mu}} \theta v D R_{C}^{0} D, S \succeq 0 \\
\tilde{D}_{X}^{f}+\tilde{D}_{S}^{f}=-\nabla \Psi(\tilde{V}),
\end{gathered}
$$

where the kernel function of $\Psi$ is given by

$$
\psi(t):= \begin{cases}\frac{t^{2}-1}{2}+\frac{t^{1-p}-1}{p-1}, & p \in[0,1) ; \\ \frac{t^{2}-1}{2}-\log (t), & p=1 .\end{cases}
$$

Since $\psi^{\prime}(t)=t-t^{-p}$, the third equation in the system (11) can be rewritten as

$$
D_{X}^{f}+D_{S}^{f}=\sqrt{1-\theta}\left(\tilde{V}^{-p}-\tilde{V}\right) .
$$

In the sequel, the feasibility step will be based on the Equation (13).

\section{Some Technical Results}

We recall some interesting results from Klerk [1]. In the sequel, we denote the iterates after a centrality step as $X^{+}, y^{+}, S^{+}$.

Lemma 2 Let $X, S$ satisfy the Slater's regularity condition and $\mu>0$. If $\delta:=\delta(X, S ; \mu)<1$, then the fullNT step is strictly feasible.

Corollary 3 Let $X, S$ satisfy the Slater's regularity condition and $\mu>0$. If $\delta:=\delta(X, S ; \mu)<1$. One has $\operatorname{Tr}\left(X^{+} S^{+}\right)=n \mu$.

Lemma 4 After a feasible full-NT step the proximity function satisfies

$$
\delta\left(X^{+}, S^{+} ; \mu\right) \leq \frac{\delta^{2}}{\sqrt{2\left(1-\delta^{2}\right)}} .
$$

Lemma 5 If $\delta:=\delta(X, S ; \mu)<1 / \sqrt{2}$, then

$$
\delta\left(X^{+}, S^{+} ; \mu\right)<\delta^{2} .
$$

The required number of centrality steps can easily be computed. After the $\mu$-update, one has $\delta:=\delta\left(X, S ; \mu^{+}\right)<1 / \sqrt{2}$, and hence after $k$ centrality steps the iterates $(X, y, S)$ satisfy

$$
\delta\left(X, S ; \mu^{+}\right) \leq\left(\frac{1}{\sqrt{2}}\right)^{2^{k}} .
$$

From this, one deduces easily that $\delta\left(X, S ; \mu^{+}\right) \leq \tau$ holds, after at most

$$
\log _{2}\left(\log _{2} \frac{1}{\tau^{2}}\right) .
$$

We give below a more formal description of the algorithm in Figure 1.

The following lemma stated without proof, will be useful for our analysis.

Lemma 6 (See [10], Lemma 2.5) For any $t>0$, one has

$$
\left|t^{-\frac{1-p}{2}}-t^{\frac{1-p}{2}}\right| \leq\left|t^{-1}-t\right|, \quad p \in[0,1] .
$$

By applying Lemma (6), one can easily verify so that 


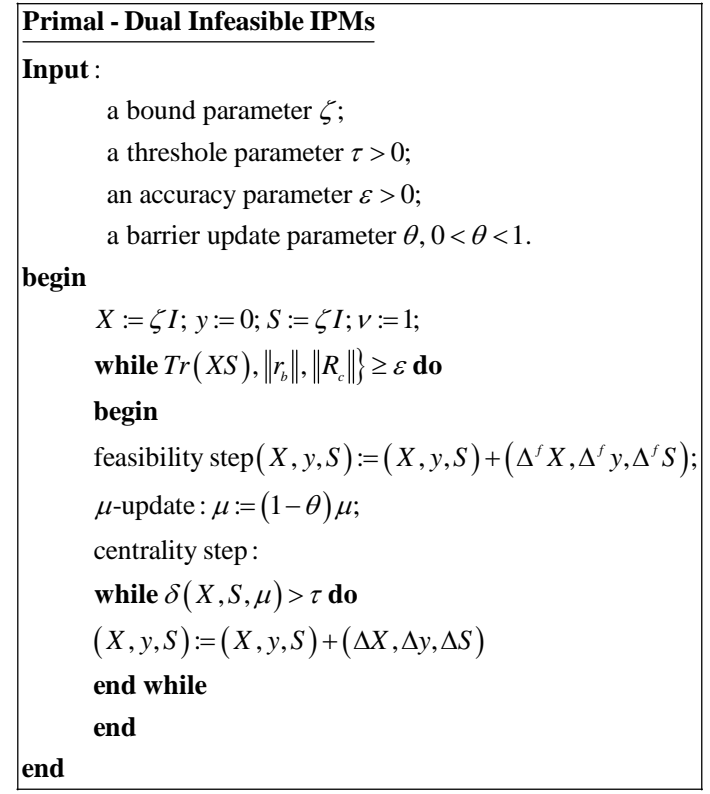

Figure 1. Primal-dual infeasible IPMs.

for any $\theta \in(0,1)$, we have:

$$
\begin{aligned}
& \left|(1-\theta)^{-\frac{1-p}{4}}-(1-\theta)^{\frac{1-p}{4}}\right| \\
& =\mid\left((1-\theta)^{\frac{1}{2}}\right)^{-\frac{1-p}{2}}-\left((1-\theta)^{\left.\frac{1}{2}\right)^{\frac{1-p}{2}}} \mid\right. \\
& \leq\left|(1-\theta)^{-\frac{1}{2}}-(1-\theta)^{\frac{1}{2}}\right|=\frac{\theta}{\sqrt{1-\theta}},
\end{aligned}
$$

and furthermore, according to (6), we obtain:

$$
\delta\left(V^{\frac{1-p}{2}}\right) \leq \delta(V) .
$$

Lemma 7 According to the result of Corollary (3), for any $p \in[0,1]$ one has

$$
\left\|V^{\frac{1-p}{2}}\right\|_{F} \leq \sqrt{n}
$$

Proof. By applying Hölder inequality and using $\|V\|_{F}^{2}=n$, we obtain

$$
\begin{aligned}
& \left\|V^{\frac{1-p}{2}}\right\|_{F}^{2}=\left\|\lambda\left(V^{\frac{1-p}{2}}\right)\right\|^{2}=\sum_{i=1}^{n} \lambda_{i}\left(V^{\frac{1-p}{2}}\right)^{2} \\
& =\sum_{i=1}^{n} \lambda_{i}(V)^{1-p} \cdot 1 \leq\left(\sum_{i=1}^{n} \lambda_{i}(V)^{2}\right)^{\frac{1-p}{2}} \cdot\left(\sum_{i=1}^{n} 1\right)^{\frac{1+p}{2}}
\end{aligned}
$$

and the result follows.

The following Lemma gives an upper bound for the proximity-measure of the matrix $\tilde{V}^{\frac{1-p}{2}}$.

Lemma 8 Let $(X, S)$ be a primal-dual NT pair and $\mu>0$ such that $\operatorname{Tr}(X S)=n \mu$. Moreover let $\delta(V)=\delta(X, S ; \mu)$ and $\tilde{V}:=V / \sqrt{1-\theta}$. Then

$$
4 \delta^{2}\left(\tilde{V}^{\frac{1-p}{2}}\right) \leq 4(1-\theta)^{\frac{1-p}{2}} \delta^{2}(V)+\frac{\theta^{2} n}{1-\theta} .
$$

Proof. Since the $(X, S)$ is a primal-dual pair and by applying Lemma (7), and the two inequalities (15) and (16), we can get:

$$
\begin{aligned}
& 4 \delta^{2}\left(\tilde{V}^{\frac{1-p}{2}}\right)=\left\|\tilde{V}^{-\frac{1-p}{2}}-\tilde{V}^{\frac{1-p}{2}}\right\|_{F}^{2}=\left\|\lambda\left(\tilde{V}^{-\frac{1-p}{2}}\right)-\lambda\left(\tilde{V}^{\frac{1-p}{2}}\right)\right\|^{2} \\
= & \left\|(1-\theta)^{\frac{1-p}{4}} \lambda\left(V^{-\frac{1-p}{2}}\right)-(1-\theta)^{-\frac{1-p}{4}} \lambda\left(V^{\frac{1-p}{2}}\right)\right\|^{2} \\
= & \|(1-\theta)^{\frac{1-p}{4}}\left(\lambda\left(V^{-\frac{1-p}{2}}\right)-\lambda\left(V^{\frac{1-p}{2}}\right)\right) \\
& +\left((1-\theta)^{\frac{1-p}{4}} \lambda\left(V^{\frac{1-p}{2}}\right)-(1-\theta)^{-\frac{1-p}{4}} \lambda\left(V^{\frac{1-p}{2}}\right)\right) \|^{2} \\
= & 4(1-\theta)^{\frac{1-p}{2}} \delta^{2}\left(V^{\frac{1-p}{2}}\right)+\frac{\theta^{2}}{1-\theta}\left\|\lambda\left(V^{\frac{1-p}{2}}\right)\right\|^{2} \\
& +2(1-\theta)^{\frac{1-p}{4}} \frac{\theta}{\sqrt{1-\theta}}\left(\lambda\left(V^{-\frac{1-p}{2}}\right)-\lambda\left(V^{\frac{1-p}{2}}\right)\right)^{T} \lambda\left(V^{\frac{1-p}{2}}\right) \\
\leq & 4(1-\theta)^{\frac{1-p}{2}} \delta^{2}(V)+\frac{\theta^{2} n}{1-\theta},
\end{aligned}
$$

since the last term in the last equality is negative. This completes the proof of the Lemma.

Lemma 9 (See [1], Lemma 6.1). If one has $\operatorname{det}(X(\alpha) S(\alpha))>0, \forall 0 \leq \alpha \leq \bar{\alpha}$, then $X(\bar{\alpha}) \succ 0$, and $S(\bar{\alpha}) \succ 0$.

Let $Q$ be an $n \times n$ real symmetric matrix and $M$ be an $n \times n$ real skew-symmetric matrix, we recall the following result.

Lemma 10 (See [7], Lemma 3.8). If $Q$ is positive definite, then $\operatorname{Tr}\left((Q+M)^{-1}\right) \leq \operatorname{Tr}\left(Q^{-1}\right)$.

Lemma 11 (See [1], Lemma 6.3). If $Q$ is positive definite, then $\operatorname{det}(Q+M)>0$.

Lemma 12 (See [7], Lemma 3.10). Let $A, B \in \mathbb{S}^{n}$, $A+B \succ 0$ and $\lambda_{\text {min }}(A)+\lambda_{\text {min }}(B)>0$. Then

$$
\begin{aligned}
& \sum_{i=1}^{n} \lambda_{i}(A+B)=\sum_{i=1}^{n}\left(\lambda_{i}(A)+\lambda_{i}(B)\right), \\
& \sum_{i=1}^{n} \frac{1}{\lambda_{i}(A+B)}=\sum_{i=1}^{n} \frac{1}{\lambda_{i}(A)+\lambda_{i}(B)} .
\end{aligned}
$$

Lemma 13 (See [11], Lemma A.1) For $i=1, \cdots, m$, let $f_{i}: R_{+} \rightarrow R$ denote a convex functions. Then, for any nonzero $\mathrm{z} \in R_{+}^{n}$, the following inequality 


$$
\sum_{i=1}^{n} f_{i}\left(z_{i}\right) \leq \frac{1}{e^{T} z} \sum_{j=1}^{n} z_{j}\left(f_{j}\left(e^{T} z\right)+\sum_{i \neq j} f_{i}(0)\right)
$$

holds.

\section{Analysis of the Feasibility Step}

\subsection{The Feasibility Step}

As established in Section 2, the feasibility step generates new iterates $X^{f}, y^{f}$ and $S^{f}$ that satisfy the Feasibility conditions for $\left(P_{v^{+}}\right)$and $\left(D_{v^{+}}\right)$(i.e., primal feasible and dual feasible), except possibly the positive semidefinite conditions. A crucial element in the analysis is to show that after the feasibility step, the inequality $\delta\left(X^{f}, S^{f} ; \mu^{+}\right) \leq 1 / \sqrt{2}$ holds, i.e., that the new iterates are within the region where the Newton process targeting at the $\mu^{+}$-centers of $\left(P_{v^{+}}\right)$and $\left(D_{v^{+}}\right)$is quadratically convergent. Let $X, y$ and $S$ denote the iterates at the start of an iteration and assume that $\delta(X, S ; \mu) \leq \tau$. Recall that at the start of the first iteration this is true since $\delta\left(X^{0}, S^{0} ; \mu^{0}\right)=0$. Defining $D_{X}^{f}$ and $D_{S}^{f}$ as in (4) and $V^{f}$ as in (3). We may write

$$
\begin{aligned}
& \left(V^{f}\right)^{2}=\frac{1}{\mu} D^{-1} X^{f} S^{f} D, \\
& X^{f}=X+\Delta^{f} X=\sqrt{\mu} D\left(V+D_{X}^{f}\right) D \\
& S^{f}=S+\Delta^{f} S=\sqrt{\mu} D^{-1}\left(V+D_{S}^{f}\right) D^{-1} .
\end{aligned}
$$

Therefore $X^{f} S^{f}=\mu D\left(V+D_{X}^{f}\right)\left(V+D_{S}^{f}\right) D^{-1}$ which implies that

$$
X^{f} S^{f} \sim \mu\left(V+D_{X}^{f}\right)\left(V+D_{S}^{f}\right) .
$$

According to (8), Equation (13) can be rewritten as

$$
D_{X}+D_{S}=\sqrt{1-\theta}\left((1-\theta)^{\frac{p}{2}} V^{-p}-\frac{V}{\sqrt{1-\theta}}\right),
$$

and by multiplying both side from the left with $V$, we get

$$
V D_{X}+V D_{S}=(1-\theta)^{\frac{1+p}{2}} V^{1-p}-V^{2} .
$$

To simplify the notation in the sequel, we denote

$$
\begin{aligned}
& D_{X S}^{f}:=\frac{1}{2}\left(D_{X}^{f} D_{S}^{f}+D_{S}^{f} D_{X}^{f}\right) \\
& M:=\left(D_{X}^{f} V-V D_{X}^{f}\right)+\frac{1}{2}\left(D_{X}^{f} D_{S}^{f}-D_{S}^{f} D_{X}^{f}\right)
\end{aligned}
$$

Note that $D_{X S}^{f}$ is symmetric and $M$ is skew-symmetric. Now we may write, using (19),

$$
\begin{aligned}
& \left(V+D_{X}^{f}\right)\left(V+D_{S}^{f}\right) \\
& =V^{2}+V D_{S}^{f}+D_{X}^{f} V+D_{X}^{f} D_{S}^{f} \\
& =(1-\theta)^{\frac{1+p}{2}} V^{1-p}-V D_{X}^{f}+D_{X}^{f} V+D_{X}^{f} D_{S}^{f} .
\end{aligned}
$$

By subtracting and adding $\frac{1}{2} D_{S}^{f} D_{X}^{f}$, to the last expression we obtain

$$
\begin{aligned}
& \left(V+D_{X}^{f}\right)\left(V+D_{S}^{f}\right) \\
& =(1-\theta)^{\frac{1+p}{2}} V^{1-p}-V D_{X}^{f}+D_{X}^{f} V \\
& +\frac{1}{2}\left(D_{X}^{f} D_{S}^{f}+D_{S}^{f} D_{X}^{f}\right)+\frac{1}{2}\left(D_{X}^{f} D_{S}^{f}-D_{S}^{f} D_{X}^{f}\right) \\
& =(1-\theta) \tilde{V}^{1-p}+D_{X S}^{f}+M .
\end{aligned}
$$

Using (20) and (17), we get

$$
X^{f} S^{f} \sim \mu\left((1-\theta) \tilde{V}^{1-p}+D_{X S}^{f}+M\right) .
$$

Note that due to (8), $\quad \tilde{V}^{1-p}$ is positive definite.

Lemma 14 Let $X \succ 0$ and $S \succ 0$. Then the iterates $\left(X^{f}, y^{f}, S^{f}\right)$ are strictly feasible if

$$
(1-\theta) \tilde{V}^{1-p}+D_{X S}^{f} \succ 0 \text {. }
$$

Proof. We begin by introducing a step length $\alpha \in[0,1]$, and we define

$$
X^{\alpha}=X+\alpha \Delta^{f} X, y^{\alpha}=y+\alpha \Delta^{f} y, S^{\alpha}=S+\alpha \Delta^{f} S .
$$

We then have $X^{0}=X, X^{1}=X^{f}$ and similar relations for $y$ and $S$. It is clear that $\operatorname{det}\left(X^{0} S^{0}\right)>0$. We want to show that the determinant of $X^{\alpha} S^{\alpha}$ remains positive for all $\alpha \leq 1$. We may write

$$
\begin{aligned}
& \frac{X^{\alpha} S^{\alpha}}{\mu} \sim\left(V+\alpha D_{X}^{f}\right)\left(V+\alpha D_{S}^{f}\right) \\
= & V^{2}+\alpha\left(D_{X}^{f} V+V D_{S}^{f}\right)+\alpha^{2} D_{X}^{f} D_{S}^{f} \\
= & V^{2}+\alpha\left(V D_{X}^{f}+V D_{S}^{f}\right)+\alpha\left(D_{X}^{f} V-V D_{X}^{f}\right)+\alpha^{2} D_{X}^{f} D_{S}^{f} \\
= & V^{2}+\alpha\left((1-\theta) \tilde{V}^{1-p}-V^{2}\right)+\alpha\left(D_{X}^{f} V-V D_{X}^{f}\right)+\alpha^{2} D_{X}^{f} D_{S}^{f} .
\end{aligned}
$$

By subtracting and adding $\frac{\alpha^{2}}{2} D_{S}^{f} D_{X}^{f}$ and $\alpha^{2}(1-\theta) \tilde{V}^{1-p}$ to the right hand side of the above equality we obtain

$$
\begin{aligned}
& \frac{X^{\alpha} S^{\alpha}}{\mu} \sim V^{2}+\alpha\left((1-\theta) \tilde{V}^{1-p}-V^{2}\right) \\
& +\alpha\left(D_{X}^{f} V-V D_{X}^{f}\right)+\alpha^{2} D_{X S}^{f}+\frac{\alpha^{2}}{2}\left(D_{X}^{f} D_{S}^{f}-D_{S}^{f} D_{X}^{f}\right) \\
& =(1-\alpha) V^{2}+\alpha(1-\alpha)(1-\theta) \tilde{V}^{1-p} \\
& +\alpha^{2}\left((1-\theta) \tilde{V}^{1-p}+D_{X S}^{f}\right) \\
& +\alpha\left(D_{X}^{f} V-V D_{X}^{f}\right)+\frac{\alpha^{2}}{2}\left(D_{X}^{f} D_{S}^{f}-D_{S}^{f} D_{X}^{f}\right) \\
& =(1-\alpha) V^{2}+\alpha(1-\alpha)(1-\theta) \tilde{V}^{1-p} \\
& +\alpha^{2}\left((1-\theta) \tilde{V}^{1-p}+D_{X S}^{f}\right)+N,
\end{aligned}
$$


where the matrix

$$
N:=\alpha\left(D_{X}^{f} V-V D_{X}^{f}\right)+\frac{\alpha^{2}}{2}\left(D_{X}^{f} D_{S}^{f}-D_{S}^{f} D_{X}^{f}\right)
$$

is skew-symmetric for all $\alpha \in[0,1]$. Lemma (11) implies that the determinant of $X^{\alpha} S^{\alpha}$ will be positive if the symmetric matrix

$$
\begin{aligned}
(1-\alpha) V^{2} & +\alpha(1-\alpha)(1-\theta) \tilde{V}^{1-p} \\
& +\alpha^{2}\left((1-\theta) \tilde{V}^{1-p}+D_{X S}^{f}\right)
\end{aligned}
$$

is positive definite which is true for all $\alpha \in[0,1]$. This means that $X^{\alpha} S^{\alpha}$ has positive determinant. By positiveness of $X^{0}$ and $S^{0}$ and continuity of both $X^{\alpha}$ and $S^{\alpha}$, we deduce that $X^{1}$ and $S^{1}$ are positive definite which completes the proof.

We continue this section by recalling the following Lemma.

Lemma 15 (See [12], Lemma II. 60). Let $\delta=\delta(V)$ be as given by (6) and $\rho(\delta):=\delta+\sqrt{1+\delta^{2}}$. Then

$$
\frac{1}{\rho(\delta)} \leq \lambda_{i}(V) \leq \rho(\delta) \text {. }
$$

The proof of Lemma (15), together with $p \in[0,1]$, makes clear that the elements of the vector $\lambda\left(V^{1-p}\right)$ satisfy

$$
\frac{1}{\rho(\delta)} \leq \lambda_{i}\left(V^{1-p}\right) \leq \rho(\delta), i=1, \cdots, n .
$$

Furthermore, by using (8) and (22), we obtain the bounds of the elements of the vector $\lambda\left(\tilde{V}^{1-p}\right)$

$$
\frac{\sqrt{1-\theta}}{\rho(\delta)} \leq \lambda_{i}\left(\tilde{V}^{1-p}\right) \leq \frac{\rho(\delta)}{\sqrt{1-\theta}}, i=1, \cdots, n .
$$

In the sequel we denote

$$
\omega(V):=\omega:=\left\|\left(\omega_{1}, \omega_{2}, \cdots, \omega_{n}\right)\right\|:=\frac{1}{2} \sqrt{\left\|D_{X}^{f}\right\|_{F}^{2}+\left\|D_{S}^{f}\right\|_{F}^{2}},
$$

where

$$
\omega_{i}:=\frac{1}{2} \sqrt{\lambda_{i}^{2}\left(D_{X}^{f}\right)+\lambda_{i}^{2}\left(D_{S}^{f}\right)}, i=1,2, \cdots, n .
$$

This implies

$$
\begin{aligned}
\operatorname{Tr}\left(D_{X S}^{f}\right) & =\operatorname{Tr}\left(D_{X}^{f} D_{S}^{f}\right) \leq\left\|D_{X}^{f}\right\|_{2}\left\|D_{S}^{f}\right\|_{F} \\
& \leq\left\|D_{X}^{f}\right\|_{F}\left\|D_{S}^{f}\right\|_{F} \leq \frac{1}{2}\left(\left\|D_{X}^{f}\right\|_{F}^{2}+\left\|D_{S}^{f}\right\|_{F}^{2}\right)=2 \omega^{2},
\end{aligned}
$$

and

$$
\begin{aligned}
\left|\lambda_{i}\left(D_{X S}^{f}\right)\right| & =\left|\lambda_{i}\left(\frac{D_{X}^{f} D_{S}^{f}+D_{S}^{f} D_{X}^{f}}{2}\right)\right| \\
& \leq\left\|\frac{D_{X}^{f} D_{S}^{f}+D_{S}^{f} D_{X}^{f}}{2}\right\|_{F} \leq\left\|D_{X}^{f}\right\|_{F}\left\|D_{S}^{f}\right\|_{F} \leq 2 \omega^{2} .
\end{aligned}
$$

Lemma 16 Assuming $(1-\theta) \tilde{V}^{1-p}+D_{X S}^{f} \succ 0$, one has

$$
\begin{aligned}
4 \delta^{2}\left(V^{f}\right) \leq & 4(1-\theta)^{\frac{1-p}{2}} \delta^{2}(V)+\frac{\theta^{2} n}{1-\theta} \\
& +\frac{\rho(1-\theta)}{(1-\theta)^{\frac{3}{2}}-2 \rho \omega^{2}}+\frac{2 \omega^{2}}{1-\theta} .
\end{aligned}
$$

Proof. Using (6), we get

$$
\delta\left(X^{f}, S^{f} ; \mu^{+}\right):=\delta\left(V^{f}\right):=\frac{1}{2}\left\|V^{f}-\left(V^{f}\right)^{-1}\right\|_{F},
$$

where $\left(V^{f}\right)^{2} \sim \frac{X^{f} S^{f}}{\mu^{+}}$.

From (21), one has

$$
\begin{aligned}
\left(V^{f}\right)^{2} & \sim \frac{\mu}{\mu^{+}}\left((1-\theta) \tilde{V}^{1-p}+D_{X S}^{f}+M\right) \\
& =\frac{(1-\theta) \tilde{V}^{1-p}+D_{X S}^{f}+M}{1-\theta} .
\end{aligned}
$$

Due to the fact that $\operatorname{Tr}(M)=0$ since $M$ is skewsymmetric and Lemma (10), we may write

$$
\begin{aligned}
4 \delta\left(V^{f}\right)^{2}= & \operatorname{Tr}\left(\left(V^{f}\right)^{2}\right)+\operatorname{Tr}\left(\left(V^{f}\right)^{-2}\right)-2 n \\
\leq & \operatorname{Tr}\left(\frac{(1-\theta) \tilde{V}^{1-p}+D_{X S}^{f}}{1-\theta}\right) \\
& +\operatorname{Tr}\left((1-\theta)\left((1-\theta) \tilde{V}^{1-p}+D_{X S}^{f}\right)^{-1}\right)-2 n \\
= & \sum_{i=1}^{n} \frac{(1-\theta) \lambda_{i}\left(\tilde{V}^{1-p}\right)+\lambda_{i}\left(D_{X S}^{f}\right)}{1-\theta} \\
& +\sum_{i=1}^{n} \frac{1-\theta}{\lambda_{i}\left((1-\theta) \tilde{V}^{1-p}+D_{X S}^{f}\right)}-2 n \\
= & \sum_{i=1}^{n} \frac{(1-\theta) \lambda_{i}\left(\tilde{V}^{1-p}\right)+\lambda_{i}\left(D_{X S}^{f}\right)}{1-\theta} \\
& +\sum_{i=1}^{n} \frac{1-\theta}{(1-\theta) \lambda_{i}\left(\tilde{V}^{1-p}\right)+\lambda_{i}\left(D_{X S}^{f}\right)}-2 n \\
\leq & \sum_{i=1}^{n} \frac{(1-\theta) \lambda_{i}\left(\tilde{V}^{1-p}\right)+2 \omega_{i}^{2}}{1-\theta} \\
& +\sum_{i=1}^{n} \frac{1-\theta}{(1-\theta) \lambda_{i}\left(\tilde{V}^{1-p}\right)-2 \omega_{i}^{2}}-2 n, \\
& \frac{(1-\theta)}{1-\theta}
\end{aligned}
$$

where we apply for the third equality, Lemma (12) whose second condition is due to the requirement (24) given below.

For each $i=1,2, \cdots, n$, we define

$$
f_{i}\left(z_{i}\right):=\frac{(1-\theta) \lambda_{i}\left(\tilde{V}^{1-p}\right)+z_{i}}{1-\theta}+\frac{1-\theta}{(1-\theta) \lambda_{i}\left(\tilde{V}^{1-p}\right)-z_{i}}-2 \text {, }
$$


It is clear that $f_{i}\left(z_{i}\right)$ is convex in $z_{i}$ if $(1-\theta) \lambda_{i}\left(\tilde{V}^{1-p}\right)-z_{i}>0$. Taking $z_{i}=2 \omega_{i}^{2}$, we require

$$
(1-\theta) \lambda_{i}\left(\tilde{V}^{1-p}\right)-2 \omega_{i}^{2}>0
$$

By applying Lemma (15), the above inequality holds if

$$
2 \omega^{2} \leq \frac{(1-\theta)^{\frac{3}{2}}}{\rho(\delta)} .
$$

By using Lemma (13), we may write

$$
\begin{aligned}
4 \delta^{2}\left(V^{f}\right) \leq & \sum_{i=1}^{n} f_{j}\left(\omega_{j}\right) \leq \frac{1}{2 \omega^{2}} \sum_{j=1}^{n} 2 \omega_{j}^{2}\left(f_{j}\left(2 \omega^{2}\right)+\sum_{i \neq j} f_{i}(0)\right) \\
= & \frac{1}{2 \omega^{2}} \sum_{j=1}^{n}\left[2 \omega _ { j } ^ { 2 } \left(\left(\frac{(1-\theta) \lambda_{j}\left(\tilde{V}^{1-p}\right)+2 \omega^{2}}{1-\theta}\right.\right.\right. \\
& \left.\left.\left.+\frac{1-\theta}{(1-\theta) \lambda_{j}\left(\tilde{V}^{1-p}\right)-2 \omega^{2}}-2\right)+\sum_{i \neq j} f_{i}(0)\right)\right]
\end{aligned}
$$

where

$$
\begin{aligned}
\sum_{i \neq j} f_{i}(0) & =\sum_{i \neq j}\left(\frac{(1-\theta) \lambda_{i}\left(\tilde{V}^{1-p}\right)}{1-\theta}+\frac{1-\theta}{(1-\theta) \lambda_{i}\left(\tilde{V}^{1-p}\right)}-2\right) \\
& =\sum_{i \neq j}\left(\lambda_{i}\left(\tilde{V}^{1-p}\right)+\frac{1}{\lambda_{i}\left(\tilde{V}^{1-p}\right)}-2\right) .
\end{aligned}
$$

Furthermore, by using Lemma (8), we get

$$
\begin{aligned}
& \sum_{i \neq j}\left(\lambda_{i}\left(\tilde{V}^{1-p}\right)+\frac{1}{\lambda_{i}\left(\tilde{V}^{1-p}\right)}-2\right) \\
& =\left(\sum_{i=1}^{n} \lambda_{i}\left(\tilde{V}^{1-p}\right)+\frac{1}{\lambda_{i}\left(\tilde{V}^{1-p}\right)}-2\right) \\
& =4 \delta\left(\tilde{V}^{\frac{1-p}{2}}\right)^{2}-\left(\lambda_{j}\left(\tilde{V}^{1-p}\right)+\frac{1}{\lambda_{j}\left(\tilde{V}^{1-p}\right)}-2\right) \\
& \leq 4(1-\theta)^{\frac{1-p}{2}} \delta(V)^{2}+\frac{n \theta^{2}}{1-\theta} \\
& -\left(\lambda_{j}\left(\tilde{V}^{1-p}\right)+\frac{1}{\lambda_{j}\left(\tilde{V}^{1-p}\right)}-2\right) .
\end{aligned}
$$

We deduce

$$
\begin{aligned}
4 \delta^{2}\left(V^{f}\right) \leq & 4(1-\theta)^{\frac{1-p}{2}} \delta(V)^{2}+\frac{n \theta^{2}}{1-\theta} \\
& +\frac{1}{2 \omega^{2}} \sum_{j=1}^{n} 2 \omega_{j}^{2}\left[\frac{(1-\theta) \lambda_{j}\left(\tilde{V}^{1-p}\right)+2 \omega^{2}}{1-\theta}\right. \\
& \left.+\frac{1-\theta}{(1-\theta) \lambda_{j}\left(\tilde{V}^{1-p}\right)-2 \omega^{2}}-2-A_{j}\right]
\end{aligned}
$$

where

$$
A_{j}:=\lambda_{j}\left(\tilde{V}^{1-p}\right)+\frac{1}{\lambda_{j}\left(\tilde{V}^{1-p}\right)}-2
$$

Hence

$$
\begin{aligned}
& 4 \delta^{2}\left(V^{f}\right)=4(1-\theta)^{\frac{1-p}{2}} \delta(V)^{2}+\frac{n \theta^{2}}{1-\theta}+\frac{2 \omega^{2}}{1-\theta} \\
& +\frac{1}{2 \omega^{2}} \sum_{j=1}^{n} 2 \omega_{j}^{2} \frac{2 \omega^{2}}{\lambda_{j}\left(\tilde{V}^{1-p}\right)\left((1-\theta) \lambda_{j}\left(\tilde{V}^{1-p}\right)-2 \omega^{2}\right)} \\
\leq & 4(1-\theta)^{\frac{1-p}{2}} \delta(V)^{2}+\frac{n \theta^{2}}{1-\theta}+\frac{2 \omega^{2}}{1-\theta} \\
& +\frac{2 \omega^{2}}{\frac{\sqrt{1-\theta}}{\rho}\left((1-\theta) \frac{\sqrt{1-\theta}}{\rho}-2 \omega^{2}\right)} \\
= & 4(1-\theta)^{\frac{1-p}{2}} \delta(V)^{2}+\frac{n \theta^{2}}{1-\theta}+\frac{2 \omega^{2}}{1-\theta} \\
& +\frac{2 \rho^{2} \omega^{2}}{\sqrt{1-\theta}\left((1-\theta)^{\frac{3}{2}}-2 \rho \omega^{2}\right)} \\
= & 4(1-\theta)^{\frac{1-p}{2}} \delta(V)^{2}+\frac{n \theta^{2}}{1-\theta}+\frac{2 \omega^{2}}{1-\theta}+\frac{\rho(1-\theta)}{(1-\theta)^{\frac{3}{2}}-2 \rho \omega^{2}} .
\end{aligned}
$$

The last equality is due to (24), which completes the proof.

Because we need to have $\delta\left(V^{f}\right) \leq 1 / \sqrt{2}$, it follows from this lemma that it suffices if

$$
4 \delta(V)^{2}+\frac{n \theta^{2}}{1-\theta}+\frac{2 \omega^{2}}{1-\theta}+\frac{\rho(1-\theta)}{(1-\theta)^{\frac{3}{2}}-2 \rho \omega^{2}} \leq 2 .
$$

Now we decide to choose

$$
\tau=\frac{1}{16}, \theta=\frac{1}{8 n} .
$$

Note that the left-hand side of (25) is monotonically increasing with respect to $\omega^{2}$. By some elementary calculations, for $n \geq 1$ and $\delta(V) \leq \tau$, we obtain

$$
\omega \leq 0.35 \Rightarrow \delta\left(V^{f}\right) \leq \frac{1}{\sqrt{2}} \text {. }
$$

\subsection{Upper Bound for $\omega(V)$}

In this section we consider the linear space

$$
\mathcal{L}=\left\{\Xi \in \mathbb{S}^{n}: \operatorname{Tr}\left(\bar{A}_{i} \Xi\right)=0, i=1,2, \cdots, m\right\} .
$$

It is clear that the affine space

$$
\left\{\Xi \in \mathbb{S}^{n}: \operatorname{Tr}\left(\bar{A}_{i} \Xi\right)=\frac{1}{\mu} \theta v\left(r_{b}^{0}\right)_{i}, i=1, \cdots, m\right\}
$$


equals $D_{X}^{f}+\mathcal{L}$ and $D_{S}^{f} \in \frac{1}{\sqrt{\mu}} \theta v D R_{C}^{0} D+\mathcal{L}^{\perp}$. We can get from Mansouri and Roos [6], the following result.

Lemma 17 (See [6], Lemma 5.11) Let $Q$ be the (unique) matrix in the intersection of the affine spaces $D_{X}^{f}+\mathcal{L}$ and $D_{S}^{f}+\mathcal{L}^{\perp}$. Then

$$
2 \omega(V) \leq \sqrt{\|Q\|_{F}^{2}+\left(\|Q\|_{F}+2 \delta(V)\right)^{2}} .
$$

Note that (27) implies that we must have $\omega \leq 0.35$ to guarantee $\delta\left(V^{f}\right) \leq 1 / \sqrt{2}$. Due to the above lemma, this will certainly hold if $\|Q\|_{F}$ satisfies

$$
\|Q\|_{F}^{2}+\left(\|Q\|_{F}+2 \delta(V)\right)^{2} \leq 4 *(0.35)^{2} .
$$
have

Furthermore, according to Mansouri and Ross, we

$$
\|Q\|_{F} \leq n \theta \rho(\delta)\left(1+\rho(\delta)^{2}\right) .
$$

Since $\delta \leq \tau=1 / 16$, we may write

$$
\|Q\|_{F} \leq n \theta \rho(\delta)\left(1+\rho(\delta)^{2}\right)=2.27 \theta n \text {. }
$$

By using $\theta=\frac{1}{8 n}$, the above inequality becomes

$$
\|Q\|_{F} \leq \frac{2.27}{8} \approx 0.28 \text {. }
$$

Because we are looking for the value that we do not allow $\omega$ to exceed and in order to guarantee that $\delta^{f}(V) \leq 1 / \sqrt{2},(28)$ holds if $\|Q\|_{F}$ satisfies $\|Q\|_{F}^{2}+\left(\|Q\|_{F}+\frac{1}{8}\right)^{2} \leq 4 *(0.35)^{2}$, since $\tau \leq 1 / 16$. This will be certainly satisfied if $\|Q\|_{F} \leq 0.43$. Hence, combining this with (29), we deduce that $\delta(V) \leq 1 / \sqrt{2}$ holds.

\subsection{Iteration Bound}

In the previous sections, we have found that, if at the start of an iteration the iterates satisfies $\delta(X, S ; \mu) \leq \tau$, with $\tau=1 / 16$, then after the feasibility step, with $\theta$ as defined in (26), the iterates satisfies $\delta\left(X, S ; \mu^{+}\right) \leq 1 / \sqrt{2}$. According to (14), at most $\log _{2}\left(\log _{2} 256\right)$ centering steps suffice to get iterates that satisfy $\delta\left(X, S ; \mu^{+}\right) \leq \tau$. So each main iteration consists of at most 3 so-called inner iterations. In each main iteration both the duality gap and the norms of the residual vectors are reduced by the factor $1-\theta$. Hence, using $\operatorname{Tr}\left(X^{0} S^{0}\right)=n \zeta^{2}$, the total number of main iterations is bounded above by

$$
\frac{1}{\theta} \log \frac{\max \left\{n \zeta^{2},\left\|r_{b}^{0}\right\|,\left\|R_{c}^{0}\right\|_{F}\right\}}{\varepsilon} .
$$

Since $\theta=\frac{1}{8 n}$, the total number of inner iterations is so bounded above by

$$
24 n \log \frac{\max \left\{n \zeta^{2},\left\|r_{b}^{0}\right\|,\left\|R_{C}^{0}\right\|_{F}\right\}}{\varepsilon} .
$$

\section{Concluding Remarks}

In this paper we extended the full-Newton step infeasible interior-point algorithm to SDP. We used a specific kernel function to induce the feasibility step and we analyzed the algorithm based on this kernel function. The iteration bound coincides with the currently best known bound for IIPMs. Future research might focuses on studying new kernel functions.

\section{Acknowledgements}

The authors gratefully acknowledge the help of the guest editor and anonymous referees in improving the readability of the paper.

\section{REFERENCES}

[1] E. de Klerk, "Aspects of Semidefinite Programming," Kluwer Academic Publishers, Dordrecht, 2002.

[2] C. Roos, “A Full-Newton Step O(n) Infeasible InteriorPoint Algorithm for Linear Optimization,” SIAM Journal on Optimization, Vol. 16, No. 4, 2006, pp. 1110-1136. doi:10.1137/050623917

[3] H. Wolkowicz, R. Saigal and L. Vandenberghe, "Handbook of Semidefinite Programming: Theory, Algorithms and Applications,” Kluwer, Norwell, 1999.

[4] Y. Q. Bai, M. El Ghami and C. Roos, "A Comparative Study of Kernel Functions for Primaldual Interior-Point Algorithms in Linear Optimization," SIAM Journal on Optimization, Vol. 15, No. 1, 2004, pp. 101-128. doi:10.1137/S1052623403423114

[5] J. Peng, C. Roos and T. Terlaky, "Self-Regular Functions and New Search Directions for Linear and Semidefinite Optimization,” Mathematical Programming, Vol. 93, No. 1, 2002, pp. 129-171.

[6] H. Mansouri and C. Roos, “A New Full-Newton Step O(n) Infeasible Interior-Point Algorithm for Semidefinite Optimization,” Numerical Algorithms, Vol. 52, No. 2, 2009, pp. 225-255. doi:10.1007/s11075-009-9270-7

[7] W. Sun and Z. Liu, "A Full-NT-Step Infeasible InteriorPoint Algorithm for sdp Based on Kernel Functions,” Applied Mathematics and Computation, Vol. 217, No. 10, 2011, pp. 4990-4999. doi:10.1016/j.amc.2010.11.049

[8] G. Gu, H. Mansouri, M. Zangiabadi, Y. Q. Bai and C. Roos, "Improved Full-Newton Step O(nL) Infeasible Interior-Point Method for Linear Optimization,” Journal of Optimization Theory and Applications, Vol. 145, No. 2, 2010, pp. 271-288. doi:10.1007/s10957-009-9634-0

[9] J. Peng, C. Roos and T. Terlaky, "Self-Regularity: A New Paradigm for Primal-Dual Interior-Point Algorithms," Princeton University Press, Princeton, 2002. 
[10] W. Sun, Z. Liu and F. Tian, “A Full-Newtonstep Infeasible Interior-Point Algorithm for Linear Programming Based on a Kernel Function," Applied Mathematics and Optimization, Vol. 60, No. 2, 2009, pp. 237-251. doi:10.1007/s00245-009-9069-x

[11] H. Mansouri and C. Roos, "Simplified O(nL) Infeasible Interior-Point Algorithm for Linear Optimization Using
Full-Newton Steps,” Optimization Methods and Software, Vol. 22, No. 3, 2007, pp. 519-530. doi:10.1080/10556780600816692

[12] C. Roos, T. Terlaky and J.-Ph. Vial, "Interior Point Methods for Linear Optimization,” 2nd Edition, Theory and Algorithms for Linear Optimization, Wiley, Chichester, 1997. 